\title{
ANÁLISIS DEL DISCURSO CONTRA EL RACISMO Y EL NEO-RACISMO EN LAS CANCIONES DEL GRUPO ZEBDA
}

\author{
Ana María Iglesias Botrán ${ }^{1}$ \\ Universidad de Valladolid
}

\begin{abstract}
RESUMEN: Las cuestiones sociales derivadas de la inmigración tales como la discriminación, el racismo y el neo-racismo se plasman en la canción francesa sobre todo desde la década de los años ochenta del siglo pasado. En los textos de las canciones se muestran desde el punto de vista de los afectados los problemas que sufren las minorías procedentes de la inmigración en Francia. Por esta razón, en este artículo realizamos un análisis discursivo exhaustivo de una selección de letras de canciones del grupo musical francés Zebda. Para ello seguimos el modelo de transitividad de Halliday (2004), así como los postulados sobre el análisis del discurso de otros lingüistas (van Dijk 1999, 2003; van Leeuwen 1996; Maingueneau 1991). El objetivo de este estudio consiste en mostrar las estrategias lingüísticas y discursivas empleadas en un discurso ideológico procedente de una minoría en busca de su visibilidad y reconocimiento social.
\end{abstract}

PALABRAS CLAVE: Canción, inmigración, racismo, Zebda, análisis del discurso.

\section{DISCOURSE ANALYSIS AGAINST THE RACISM AND NEO-RACISM IN ZEBDA'S SONGS}

ABSTRACT: Social issues arising from immigration such as discrimination, racism and neo-racism is reflected in the French song especially since the decade of the eighties of last century. In the texts of the songs appear from the point of view

1. La autora es profesora del Departamento de Filología Francesa y Alemana de la Universidad de Valladolid. Este artículo pertenece a la investigación financiada por la Consejería de Educación de la Junta de Castilla y León, en el marco del proyecto titulado "40 años de comunicación musical. La función social de la canción popular en España, Francia, Reino Unido e Irlanda. Un análisis lingüístico" (VA084B11-1) concedido por orden EDU 1174/2010; publicada en el B.O.C.Y.L. con fecha 29/03/2011. 
of the problems that affected minorities of immigrant origin in France. For this reason, in this paper we present a comprehensive discourse analysis of selected lyrics French music group Zebda. To do this we follow the model of transitivity of Halliday (2004), and the postulates on discourse analysis of other linguists (van Dijk 1999, 2003, van Leeuwen 1996; Maingueneau 1991). The aim of this study is to show linguistic and discursive strategies employed in an ideological discourse from a minority seeking their visibility and social recognition.

KEYWORDS: Song, immigration, racism, Zebda, discourse analysis.

\section{Introducción}

La République, pour autant, ne traite pas encore également tous ses enfants. La majorité de ceux de l'immigration subissent toujours la ghettoïsation, les discriminations dans l'accès à la formation, à l'emploi, à la santé et à la culture, ainsi que l'humiliation du racisme et d'une répression ciblée. Autant de signes d'une société «postcoloniale », au sens où les méfaits de l'impérialisme français ont marqué les pays et leurs mentalités de part et d'autre de la Méditerranée (Lefebvre 2006: 57).

La inmigración se entiende como la entrada y permanencia temporal o definitiva en un país de personas no autóctonas, y por el término inmigrante se entiende: "aquella persona establecida en un país por la inmigración" (Diccionario Hachette). En Francia, las experiencias personales de los inmigrantes al llegar al seno de la sociedad francesa se plantean de forma ineludible vinculadas al fenómeno del rechazo social, político e institucional de estas minorías² (Ferréol y Jucquois 2004: 209-210; Semprini 1997: 30). Todas aquellas cuestiones relativas a la inmigración se plasman en diferentes manifestaciones artísticas y también en la canción. Estas expresiones suponen un reflejo de la importancia de la llegada de miles de personas de múltiples nacionalidades al territorio francés durante más de un siglo, un hecho que supuso profundas modificaciones en el seno de la sociedad francesa.

La inmigración forma parte de la historia del país galo y constituye un proceso largo y complejo que ha influido en el desarrollo económico, social, político, edu-

2. Desde el punto de vista sociológico, un grupo constituye una minoría cuando sus miembros poseen una identidad socialmente desvalorizada y considerada como inferior. La noción se refiere a una situación de desventaja relativa, ya sea demográfica, política, económica o cultural. Estas minorías son por definición más vulnerables frente a actitudes racistas o xenófobas. Por esta razón, las minorías reaccionan en ocasiones generando afirmaciones de identidad públicas y visibles, así como formas de acción colectiva (Ferréol y Jucquois 2004: 209-210; Semprini 1997: 30). 
cativo, cultural y urbanístico desde finales del siglo XIX hasta la actualidad. Esto supone un aumento lento pero progresivo del protagonismo de los inmigrantes y sus descendientes en la sociedad, así como el surgimiento de dificultades derivadas de sus circunstancias sobre todo en los momentos de crisis económicas. Se puede observar que estos movimientos migratorios están ligados a políticas de acogida o de rechazo dependiendo en cada momento de los intereses económicos de Francia. Por consiguiente, se establecen a lo largo de la historia diferentes resoluciones legislativas que permiten prácticas de discriminación laboral y económica hacia los inmigrantes (Ambrosi 2004, Noiriel 2006, Tapinos 1993, Temime 1999, Winock 2003).

Además de las diferencias profundas que provoca la segregación de las minorías en el ámbito laboral y económico, existe también lo que los sociólogos consideran neo-racismo (Ferréol y Jucquois 2004: 255-263), que se entiende como un racismo diferente al que define Lévi-Strauss, de carácter biológico:

Le racisme est une doctrine qui prétend voir dans les caractères intellectuels et moraux attribués à un ensemble d'individus, de quelque façon qu'on le définisse, l'effet nécessaire d'un commun patrimoine génétique (Lévi-Strauss 1983: 15).

Este neo-racismo no cuenta con referencias explícitas a una diferencia de raza, sino que se centra sobre la identidad cultural más que sobre las categorías raciales, y sobre la diferencia grupal más que sobre la desigualdad racialmente determinada. Desde los años ochenta del siglo pasado se forja la expresión de un neo-racismo diferenciador y cultural que designa específicamente la configuración ideológica emergente que surge, por ejemplo y en este caso concreto, del racismo contra los inmigrantes. Se trata de una xenofobia interna que interpreta una especie de nación dentro de la propia nación. Esta xenofobia evoca la existencia de una comunidad minoritaria que se presupone inasimilable ${ }^{3}$ y peligrosa para la sociedad, ésta última percibida como mayoritaria y normal. Este racismo contra los inmigrantes se presenta como una heterofobia interna y selectiva que no tiene en cuenta las cuestiones de raza o de desigualdad. De modo que, lo que se reprocha a determinadas categorías de inmigrantes es, por un lado, el hecho de ser culturalmente inasimilables y, por otro, el encarnar una amenaza de desorden para el grupo nacional, configurado en desfiguración, desnaturalización y desintegración (Ferréol y Jucquois 2004: 255-126).

3. La asimilación se entiende como el proceso por el que los inmigrantes se funden con la sociedad a la que llegan y pierden sus rasgos de identidad específica (Erba 2007: 31). 
En todo caso, las ideologías y prácticas racistas y neo-racistas buscan principalmente mantener a los otros en una situación de inferioridad, ya sean extranjeros, minorías o individuos con características diferentes a las nuestras. Esta localización puede ser simbólica pero también real. De manera especial, se sitúa al grupo objeto del racismo abajo y fuera: fuera de nuestro país, nuestra ciudad, nuestro vecindario, nuestra calle, nuestra familia, nuestros trabajos, nuestras casas. Si a estos grupos se les concede el acceso o la admisión será tan sólo en una posición inferior: en alguna otra parte siempre peor de la ciudad, en peores casas, peores trabajos, etc. (Lakoff y Johnson 1980: 50-54, 69; van Dijk 1999: 202).

La discriminación se define por lo tanto como el hecho de seleccionar excluyendo o dar trato de inferioridad a una persona o colectividad por motivos raciales, religiosos, políticos, etc. (RAE). En Francia se reconocen diecisiete tipos de discriminación basados en el origen, sexo, situación de la familia, apariencia física, el origen del apellido, el estado de salud, las minusvalías, las características genéticas, las costumbres, la orientación sexual, las ideas políticas, la edad, las convicciones religiosas, las actividades sindicales, y la pertenencia o no, verdadera o supuesta a una etnia, una nación o una raza (Erba 2007: 56). La asociación de la inmigración al racismo (Lévi-Strauss 1983: 15), al neo-racismo (Ferréol y Jucquois 2004: 255-126), así como la exclusión basada en el poder económico se denuncia de forma continua en las canciones del grupo musical Zebda y lo hace empleando determinadas estrategias discursivas que pretendemos mostrar en este estudio.

Los integrantes del grupo Zebda son en su mayoría ciudadanos franceses de segunda o tercera generación procedentes de la inmigración que han nacido y crecido padeciendo las circunstancias de los grupos denominados minorías. Su carrera musical comienza en la década de los años ochenta y, desde entonces y hasta su separación en 2007, han sido muchos los éxitos cultivados en sus cinco trabajos discográficos y en otros discos publicados junto con diferentes artistas. La experiencia comprometida de este grupo musical no se limita sólo a las letras de sus canciones y a la escena artística, sino que también se acerca a la actividad política propiamente dicha. De esta forma, en 2001 presentan una candidatura a la alcaldía de Toulouse a través de una plataforma ciudadana llamada Motivé-e-s. Consiguen pasar a la segunda vuelta en la que son derrotados frente a un partido de derechas. Sin embargo, es necesario señalar que sus canciones son en sí misma una defensa de los derechos de la ciudadanía francesa, de los valores republicanos promovidos por Francia y de una llamada a la reflexión a través de la palabra que rechaza de forma rotunda la violencia (Marx Scouras 2005: 130-187). 
Para este estudio, si bien se analizan en concreto dos canciones que se centran de forma especial en las cuestiones relativas a la discriminación, el racismo y el neo-racismo, es necesario indicar que hay otras en las que estos tópicos se repiten de manera reiterada, ya sea de forma directa o indirecta, mostrando así la protesta y el rechazo a la discriminación. Tales canciones son, por ejemplo, "La France" (1992), "Héreditaire" (1995), "Le bruit et l'odeur" (1995), "On est chez nous" (1998), y “J'y suis, j'y reste" (2002), entre otras. De hecho, el origen mismo del grupo musical Zebda, de sus ideas sobre la cultura francesa, de sus realidades personales dentro del ámbito francés surge de forma ineludible como resultado de los procesos de inmigración y de sus consecuencias tanto positivas como negativas. No obstante, para este análisis se han seleccionado dos canciones, "L'erreur est humaine" (2002) y "Tombés des nues" (1998), que tratan de forma clara y explícita esta cuestión.

Para realizar el análisis de las canciones emplearemos La gramática sistémicofuncional de Halliday y su modelo de análisis llamado Transitividad (2004). Este enfoque explica las estructuras lingüísticas siguiendo la idea de que el lenguaje tiene una función determinada en un contexto concreto, y que en los textos orales o escritos se representan personas que se relacionan entre sí. Además, gracias a la Transitividad (Halliday 2004: 170), se establecen diferencias entre los tipos de procesos verbales. Se puede comprobar cómo se representa en los textos que estas personas hacen cosas, crean cambios, expresan comportamientos, ven, sienten, piensan, hablan, tienen una identidad y unas características propias. La Transitividad es por lo tanto un recurso gramatical capaz de construir el flujo de la experiencia en términos de un proceso que se realiza gramaticalmente en una oración (Ghio y Fernández 2008: 93).

Siguiendo este planteamiento, hemos dividido el análisis en dos pasos. Primero analizaremos las personas representadas en las canciones con el objetivo de observar los pronombres y los sintagmas nominales con los que se representa a la primera persona, la segunda, la tercera y la audiencia. Para estudiar las personas representadas y su función dentro de los textos de las canciones seguiremos los planteamientos de Van Leeuwen sobre la representación de los actores sociales en el discurso (1996). En segundo lugar, estudiaremos la Transitividad a través los tipos de procesos y el co-texto en el que se insertan y en este caso se seguirán los postulados de la gramática-sistémico funcional de Halliday (2004).

Los procesos que aparecen en los textos de las canciones están determinados por el tipo de actividad al que el verbo hace referencia. Halliday presenta seis tipos de procesos: los procesos materiales expresan lo que los actores hacen a través de 
actos y sucesos concretos y abstractos (Halliday 2004: 179). Los procesos mentales exponen las experiencias del mundo de las personas representadas desde la percepción, cognición y afectividad (Ibid.: 197). Los procesos relacionales expresan lo que las personas representadas son y lo que tienen, de tal forma que los verbos être y avoir son los procesos relacionales típicos. Los procesos de comportamiento describen los actos fisiológicos y psicológicos, aunque ya adelantamos que la presencia en estos textos analizados es nula. Por su parte, los procesos verbales se sitúan en un lugar intermedio entre los procesos de relación y los procesos mentales y representan procesos del decir, se trata por tanto de lo que las personas representadas en los textos dicen. Finalmente, los procesos existenciales expresan las realidades inmutables vinculadas con las personas representadas o con otros elementos del co-texto. Éstos se asemejan a los relacionales ya que cuentan con un participante implicado en un proceso de ser o existir. Sin embargo, se diferencian de ellos al solo tener un participante, denominado existente, normalmente expresado en francés con Il y $a$, o Il fait.

\section{Títulos y tópicos de las canciones}

El título de la canción Tombés des nues está formulado mediante un proceso material expresado con un participio pasado y un adjunto circunstancial de lugar o procedencia en el que se ha omitido de forma intencionada el sujeto. Esta omisión tiene dos funciones. Por un lado, puede explicarse como gancho (Adam y Bonhomme 1997: 73-84) para llamar la atención del destinatario. Por otro, el sujeto elíptico se interpreta también como una forma de generalización al no aparecer de manera explícita, ya se puede interpretar que el sujeto sea nous, vous, o ils-elles. Esto supone dejar a la imaginación del destinatario la identidad del posible sujeto y que se vea obligado a acudir a la canción para complementar la información que falta. De manera que el gancho pretende que el destinatario se pregunte sobre qué personas han estado o están "tombés". En la actualidad, se utiliza el término nuages para referirse a las nubes en plural y nues ha quedado para emplearse en un registro cuidado, literario o bien para esta expresión fijada. En todos los textos de las canciones se mezclan términos de diferentes registros e idiomas, y este es un ejemplo más. Es una forma de conectar con una audiencia amplia entre la que están los jóvenes de las cités aunque también otro tipo de público.

Este título está tomado de una expresión lexicalizada originaria del siglo XVII que procede de la visión sobrenatural que en tiempos anteriores se tenía de las nubes, consideradas misteriosas por no saberse lo que podían estar escondiendo y qué podría caer de ellas. Este significado ha ido evolucionando y en la actualidad 
significa quedarse pasmado o sorprendido. Así se introduce la idea que se desarrolla a lo largo de la canción en la que se relata la experiencia de un locutor que llega a Francia con unas ideas sobre el país y lo que se va a encontrar, pero que se ve obligado a enfrentarse con una realidad que rompe con todos sus sueños:

Et les pieds nus et la tête dans les nuages ${ }^{4}$,

Le coeur au chaud et je faisais semblant,

Mais y avait pas de quoi en faire un fromage,

Au pays du Mont Blanc.

En la canción se repite constantemente je suis venu, un proceso material empleado para completar el significado del título en el que se expresa un cambio debido al movimiento y que formula el desconcierto del extranjero al verse rechazado:
J' suis venu mais je le dis avec quel air
Qu'on me reçut à reculons.
On peut pas dire mon cher Léon,
Que ce soit sur un air d'accordéon.

La sorpresa del locutor surge ante la propia República francesa que muestra al mundo ser una sociedad con proyectos basados en la igualdad y la democracia, donde los individuos se reconozcan como ciudadanos sin distinción de raza o religión (Erba 2007: 29). Sin embargo, esta imagen proyectada no se corresponde con la realidad. Por esta razón, el locutor considera que es más bien todo lo contrario. Es sabido que la uva y los vinos franceses tienen un importante prestigio internacional, al igual que Francia como país republicano, por esta razón estos dos emblemas son los elegidos para jugar metafóricamente con esta idea. Así, el locutor expresa que tenía unas determinadas expectativas antes de llegar a Francia que se desvanecen al descubrir la realidad in situ: "J'attendais le raisin mais de ces fruits locutor n'ai vu que les lignes".

En la canción L'erreur est humaine se expresa mediante un proceso relacional intensivo una afirmación tomada de una expresión lexicalizada. En este título, el

4. Señalamos el juego de sonidos entre los términos nus que significa "desnudos", nues, y nuages, ambos términos con el mismo significado "nubes". Este juego puede completarse por la similitud de sonidos con la designación pieds noirs. De igual forma se reescribe aquí la expresión avoir la tête dans les nuages. Una frase que condensa todo lo que se explica en este epígrafe, que se centra en el choque de la idea que se tiene de Francia antes de llegar, idealizada e irreal, con la Francia que realmente se encuentran, que les discrimina y maltrata. 
sujeto de la oración es un nombre común que se refiere a una realidad abstracta: "l'erreur" y el actor real de la acción aparece como un complemento. El significado cambiaría si se atribuyeran las acciones correspondientes a cada rango de la oración, esto es, hubiera sido distinto decir: "l'humain fait l'erreur". Sin embargo, el sustantivo aparece asociado a un proceso relacional, lo cual indica una omisión del proceso material y de actor o causante del error. De tal forma, se presenta como un proceso natural, inevitable, general, no cuestionable porque de forma habitual es así, lo que conlleva ausencia de espíritu crítico y de una posibilidad de cambio, mejora o restructuración. De igual manera, el hecho de emplear una frase ya fijada en la lengua enuncia una realidad estereotipada y generalizadora que esconde las particularidades individuales. El gancho de este título viene suscitado por un artículo definido "la/l" " y el sustantivo "erreur" que puede hacer pensar al destinatario cual es el error del que se está hablando, o bien, las razones por las que el error es algo inherente al ser humano.

No obstante, cuando se aborda la canción entera y se estudia sobre todo el estribillo, se matiza y se critica el significado del título: "L'erreur est humaine, mais l'humain?". Con la interrogación retórica se marca de forma explícita que la utilización de una frase hecha como la del título, que de alguna manera legitima los errores o incluso los defiende por formar parte de la propia esencia humana, no es una razón que en la actualidad pueda servir como explicación a determinadas aberraciones cotidianas, como son el caso de la exclusión, el neo-racismo y el rechazo a la diferencia.

El texto completo de la canción matiza el contenido y se refiere a personas procedentes de diferentes países de África, con diferentes orígenes culturales y religiosos:

Il était d'où, il était Jah, il était Djinn 5 .

Voici l'histoire de celui qui portait pas de jean,

Mais des tissus bariolés de l'Afrique,

Des sandalettes de plastique...

Estas personas conviven de forma cotidiana con los prejuicios y las prácticas discriminatorias de la sociedad, incidiendo sobre todo en las procedentes de la policía:

5. Jah es la abreviación de Jehová, que significa Dios en la lengua hebraica. Djinn significa "genio" en árabe y se refiere a un ser fabuloso con figura humana, que interviene en cuentos y leyendas orientales. 
L'histoire d'un pays qui raconte aux enfants,

Que c'est nous les méchants! (...).

Un pays qui veut nous protéger des malheurs,

Mais (il) veut être le gendarme

Et c'est nous les voleurs.

Además de esta crítica, extendida a lo largo del texto de la canción, se incide también sobre las formas de neo-racismo por las que se discrimina y se estigmatiza a las minorías en situación económica precaria: "Nous on savait que si la thune a pas d'odeur, pas la sentir c'est passer un mauvais quart d'heure". En este caso se recurre a la intertextualidad (Maingueneau 1991: 154) para hacer referencia también al título del disco y de la canción Le bruit et l'odeur, ambos inspirados en un discurso de Jacques Chirac ${ }^{6}$. La cuestión del olor aparece a menudo unida al dinero, refiriéndose a aquellas minorías o individuos que tienen fama, dinero y éxito en sus carreras profesionales y a las que la sociedad francesa no rechaza ni discrimina precisamente por el hecho de situarse en otra situación económica. Por esta razón procede citar en este caso la afirmación de Magyd Cherfi:

La France aime Zizou, pas les Arabes:

La France aime Khaled, pas les Algériens;

La France aime Zebda, pas les beurs.

(Cherfi 2004: 26).

\section{Personas representadas: primera y tercera persona, audiencia}

La primera persona del endogrupo locutor aparece de forma repetida en las dos canciones aunque su presencia es mayor en "Tombés des nues", lo que indica un interés por mostrar que es esta persona la que toma la palabra y cuenta su visión de los hechos. En ambos casos se refiere a un locutor, sin embargo, éste va alter-

6. Nos referimos a un discurso proferido por Jacques Chirac, el entonces presidente de la República Francesa, en un acto público ante los miembros de su partido: "Comment voulez-vous, que le travailleur français qui travaille avec sa femme et qui ensemble gagnent environ $15000 \mathrm{FF}$, et qui voit sur le palier à côté de son HLM entassée, une famille avec un père de famille, trois ou quatre épouses et une vingtaine de gosse et qui gagne $50000 \mathrm{FF}$ de prestation sociale, sans naturellement travailler. Si vous ajoutez à cela le bruit et l'odeur, eh bien ! Le travailleur français sur le palier, il devient fou ! Et ce n'est pas être raciste que de dire cela. Nous n'avons plus les moyens d'honorer le regroupement familial et il faut enfin ouvrir le débat qui s'impose dans notre pays, qui est un vrai débat moral, pour savoir si il est naturel que les étrangers puissent bénéficier au même titre que les Français d'une solidarité nationale à laquelle ils ne participent pas, puisqu'ils ne payent pas d'impôts" (Chirac 1991). 
nando su presencia con nous y on: en primer lugar aparece el locutor en primera persona del singular: "Je suis venu", y en el estribillo surge el on: "on est jamais invité quand on est sans thune, sandales ${ }^{7}$ ou même sans papiers". Se presenta así al endogrupo en términos de generalización asociado a un proceso material negativo que se enfatiza con el adjunto circunstancial temporal "jamais". La aliteración del sonido /sã/ -sans- ayuda a llamar la atención sobre las ausencias materiales que determinan las razones de la exclusión. La primera persona aparece representada como personas y en referencia a un contexto estrechamente vinculado a los africanos inmigrantes en Francia. Se trata por tanto de la técnica de personalización y de especialización respectivamente (van Leeuwen 1996: 59), cuyo objetivo es acercar estas personas a la audiencia.

La presencia del endogrupo en el texto se enfatiza también gracias a sintagmas nominales que expresan los vínculos con posesivos: "mon peuple à moi, mon nom, mon visage". Es necesario señalar la importancia del hecho de evocar el rostro asociado a la primera persona, puesto que es la manera por la que las personas se reconocen. Es en esta dimensión donde el individuo aparece como único y es la forma en la que se hace visible para el otro (Lévinas en Ferréol y Jucquois 2004:6). Se habla de la importancia del rostro y del nombre ya que ambos son los elementos que identifican de manera individual y única a las personas, por esta razón se enuncian en el texto de manera explícita. Se refleja así el concepto de alteridad entendido como el encuentro y desencuentro de la persona y el otro. Siguiendo esta misma idea y recurriendo a términos hegelianos, surge aquí el reconocimiento del otro como parte de sí mismo, esto es: "ser uno mismo en el otro". En este caso concreto, el locutor no se ve reconocido por la mirada del otro (Andrés 2007: 114; Férreol y Jucquois 2004: 4-7). Además, se trata de una metonimia en la que se toma la parte por el todo, ya que, en este caso, la metonimia funciona cuando se percibe a la persona en términos de su cara y el resto de personas actúa y reacciona en función de esas percepciones (Lakoff y Johnson 1986: 75).

En todo el análisis de las canciones se puede constatar que los pronombres tienen una función deíctica cuyo fin es conceptualizar la identidad de grupo, las coaliciones, las partes, los pertenecientes al grupo y los que no lo son (Chilton 2004: 56). Sin embargo, en algunos casos, los pronombres puede tener diversas

7. Homofonía de: sandale-sans dalle. La primera se refiere a no tener calzado, remitiendo a los pies desnudos (Pies nus). No puede probarse de forma rigurosa, pero quizás con esta homofonía se pretende evocar la expresión avoir la dalle. 
funciones con las se pueden captar la atención de la audiencia, como veremos a continuación.

En la canción L'erreur est humaine, la primera persona difiere de los enfoques teóricos, puesto que la canción comienza hablando de una tercera persona $\mathrm{il}$, celui qui: "Voici l'histoire de celui qui portait pas de jean (...) il vivait là au milieu des bâtiments". En este caso, si se aplicara un enfoque estrictamente gramatical se consideraría esta persona representada por el pronombre $i l$ excluida de la relación de la elocución (Kerbrat-Orecchioni 1980: 41-43), alejada del centro deíctico y sin participar de forma directa en la relación de las personas representadas cercanas al centro deíctico, sino más bien al contrario, muy distante con respecto a ellas (Yule 1996: 11). Sin embargo, al observar el co-texto y al continuar con el análisis de las demás personas, se observa que, en primer lugar, se pasa del empleo del il al pronombre nous: "Que c'est nous les méchants!", después se enuncia con procesos verbales lo que la tercera persona del singular dice, y a continuación se emplea el on y finalmente el locutor:

\footnotetext{
On sera plus que des poules qui picorent (...).

On savait que c'était une semaine (...).

En vérité, je vous le dit, y sont dedans (...).

Mon peuple à moi a bien plus de mille ans.
}

Esto muestra que en el texto se pasa de la presentación de la persona $i l$ como excluida del grupo, a ser entendida como la primera y situarse dentro del nous o endogrupo. Esto indica la voluntad del emisor por acercar e incluir a las personas representadas en un grupo. A continuación, finaliza con el pronombre inclusivo on, cuya función sería identificar al locutor con sus iguales, situados en este caso en la audiencia, así como enfatizar la importancia del endogrupo (Maingueneau 1991: 111-112). Asimismo, se pasa de la tercera persona a la primera haciendo que los argumentos relatados aparentemente lejanos sean en realidad los mismos del endogrupo. Se logra de esta forma la fusión de todas las personas con el objetivo de, por un lado, tratar de que todas las personas que padecen los mismos males se identifiquen en un primer momento a través del $i l y$, por otro, mostrar la pertenencia al grupo así como la solidaridad con éste. De igual forma, la intención de esta estrategia discursiva pretende también que la audiencia se identifique con las personas representadas, o bien dar a conocer los hechos para que la audiencia sienta la solidaridad del endogrupo en el caso que proceda a través de lo que se relata en la canción.

Estas estrategias en las que se van combinando las personas y en las que se explica lo que son, lo que hacen o dicen, indican un alto nivel de especificación 
del endogrupo. Para ello se emplean referencias concretas que pasan a ser generales para mostrar así los problemas comunes de un grupo a nivel general pero sin olvidar las individualidades específicas e identificables (van Leeuwen 1996: 46). De tal forma que, retomando los términos hegelianos, se llega al conocimiento desde la afirmación del locutor de lo particular en lo general. Ello implica un proceso que viabiliza por un lado la comprensión de sí mismo, y por otro el reconocimiento mutuo entre los individuos, y por lo tanto, posibilita la reconciliación colectiva (Andrés 2007: 118): "Il vivait là, au milieu des bâtiments, et racontait à tue-tête des boniments, l'histoire d'un pays qui raconte aux enfants, que c'est nous les méchants!'. En estos casos en los que aparecen personas il que en realidad expresan la presencia de un grupo nous se estaría aplicando la asimilación por individualización (van Leeuwen 1996: 48) que se realiza a través de los ejemplos singulares como los que se dan en esta canción, que habla de una persona que vive en uno de los edificios, que dice que los franceses describen y perciben a los inmigrantes nous- como "méchants". Esta estrategia señala un alto nivel de especificación que trataría de mostrar a través de la ironía una auto-representación positiva del endogrupo (van Dijk 2003: 60-63). Se trata de una expresión doble en la que no se dice lo que realmente se piensa, sino lo contrario, con el fin de mostrar que los estereotipos en muchas ocasiones no muestran la realidad (Moura 2010: 12)

Sin embargo, aparece aquí una excepción a la teoría de la distancia del il (Kerbrat-Orecchioni 1980: 41-43; Yule 1996: 11), ya que también se representa con el ils la tercera persona externa: "Avant qu'ils se ramènent ou qu'y nous crèvent, avant qu'ils nous pompe l'oxygène et la sève". La tercera persona alejada del centro deíctico y que establece la relación dialógica con el endogrupo aparece por tanto de forma manifiesta, aunque con un bajo grado de especificación. Esta estrategia de indeterminación (van Leeuwen 1996: 51) se realiza con el pronombre de tercera persona ils sin concretar, nombrar ni especificar la identidad de esta tercera persona. El mayor grado de indeterminación surge con el empleo de on referido a la tercera persona: “(il) paraît qu'ici on ne boit que du vin; qu' on avait peur des voisins". Esta técnica se denomina asimilación por colectivización (van Leeuwen 1996: 48-50) y consiste en presentar esta persona con un pronombre para crear una persona-grupo general, difusa y poco caracterizada.

En las pocas referencias que se hace al exogrupo se emplea la categorización por funcionalización con un conjunto de nombres y referencias que denotan lugares u objetos vinculados (van Leeuwen 1996: 54). En este caso, el espacio se presenta con un pronombre y una metonimia que expresa una parte para referirse a un todo. Es decir, se habla del Mont Blanc, de la costa y el mar, del vino y del acordeón, 
todos ellos como partes señaladas de Francia, que es el todo: "Au pays du Mont Blanc; Le soleil ou le bord de la mer; paraît qu'ici on ne boit que du vin, que ce soit sur l'air d'accordéon". Francia se representa por tanto segmentada y reducida a elementos que forman parte sobre todo de sus estereotipos más conocidos.

En el caso de la canción L'erreur est humaine se dice: "il (le pays) y veut être le gendarme; ils vous ferait manger du sable; ils nous font marcher sur la tête; ils nous crèvent". En esta cita se observa que entra la tercera persona del exogrupo y el endogrupo se establece una relación en términos de pasivización (van Leeuwen 1996: 44). El actor social pasivizado "nous" padece el proceso material realizado por el exogrupo ils. Se trata de una forma de enfatizar la acción realizada (van Leeuwen 1996: 59).

La pasivización es una técnica por la que las personas representadas en un grupo aparecen reflejadas como víctimas que padecen la agresión de otro grupo. En este caso el agresor es el exogrupo ils y la víctima el endogrupo nous. Se critica así de forma explícita a la tercera persona, y se establece una relación dialógica entre nosotros y ellos, como se explica en el llamado cuadrado ideológico de van Dijk (2003: 60-63), que consiste en enfatizar los aspectos positivos del endogrupo y los negativos del exogrupo a través de diferentes niveles de especificación. De tal forma, se enfatiza la presencia de las primeras personas locutor, nous, on y se sitúan los hechos, una estrategia muy común en la explicación histórica (Achugar 2008: 61). Por medio de estas estrategias, en todo momento la primera persona aparece como una víctima que sufre las acciones del exogrupo, al mismo tiempo que evade todas sus posibles responsabilidades, de tal forma que se asigna toda la responsabilidad a la tercera persona y se omiten las que pudiera tener la primera. Se crea así una memoria individual con ánimo de ser colectiva que se transmite a través de la canción y que configura a nivel discursivo un pasado histórico que pretende ser objetivo. Sin embargo, éste muestra sólo la visión de la primera persona que toma la palabra. No se presentan de forma real las dos ópticas de lo que realmente sucedió, sino que sólo se muestra la del endogrupo. Así, la tercera persona ils aparece silenciada, lo que favorece su imagen de ser la causante de todos los males del endogrupo, convirtiéndose así en un símbolo de ataque, agresora, discriminadora y germen de la injusticia, siempre asociado al país, a la historia. Esta representación lleva a pensar que la realidad que se presenta fue un error: "Un pays qui", "L'erreur", "L'histoire”. Se trata por tanto de una definición de la identidad del endogrupo en oposición al otro, que conlleva una relación dialógica basada en la alteridad, y en la percepción de unos y otros por parte del locutor. 
Al no existir un mismo nivel de especificación en ambos grupos, se muestra la inclinación del discurso hacia los intereses del endogrupo, lo que confiere a estos textos un alto nivel de subjetividad y por lo tanto, en rigor, poca credibilidad histórica. El hecho de emplear en las canciones técnicas discursivas de los discursos ideológicamente marcadas le hace perder al texto su carácter objetivo, y ser un discurso subjetivo y manipulado en su propio beneficio e interés. Todo ello indica una vez más que la representación de los actores y de los acontecimientos expresan el significado ideológico y experiencial de los textos, y que a través de ellos se construye una versión concreta del pasado (Achugar 2008: 55).

En el conjunto de canciones de este macrotema la audiencia aparece de forma escasa, y en el caso de la canción Tombés des nues se registra una única aparición. En la canción L'erreur est humaine, la audiencia aparece en cuatro ocasiones, dos de ellas asociada a procesos verbales: "Méfie-toi, je vous le dit", en una a un proceso mental: "Quand tu y penses", y la última expresada con un proceso relacional: "Tu es libre". Con estos ejemplos se puede observar que el emisor se dirige a la audiencia con la intención de enfatizar que lo que el locutor dice es verdad, y pidiéndole que reflexione de forma libre. Se insiste en la condición de libertad de pensamiento de la audiencia porque, al dar una versión subjetiva del pasado, se solicita que se reflexione sobre los demás posibles discursos sobre los mismos hechos. Así, el texto enuncia una realidad de forma subjetiva, justificando esta versión pedir a la audiencia que desconfíe de todos los discursos subjetivos y que decida por ella misma su propio punto de vista sobre los hechos, de ahí el imperativo: "Méfie-toi". En todo caso, estas apelaciones a la audiencia tratan de captar su atención con el objetivo de hacer reflexionar sobre lo que se relata.

\section{Transitividad: procesos materiales, procesos mentales, procesos verbales, procesos de comportamiento y procesos relacionales}

En las canciones analizadas en este macrotema se observa que los procesos materiales, relacionales y verbales son los que cobran mayor protagonismo.

En relación con los procesos materiales, hay que destacar la repetición hasta siete veces de: "Je suis venu". Esta insistencia en el hecho de venir al país indica en la canción Tombés des nues que el locutor se reconoce sin reservas como inmigrante, auto-afirmando de esta forma su propia identidad y reconociendo que el hecho de venir se hizo con convicción, de forma voluntaria, con unas ideas excelentes del país que se iba a encontrar: "Je suis venu mais locutor le dis avec quel air, qu'on me reçut à reculons". 
En el ejemplo de la canción L'erreur est humaine: “Avant qu'ils nous pompe l'oxygène et la sève au nombre des thunes (...), au nom du $\mathrm{Nasdaq}^{8}$, au nom de bénéfice". Se emplean procesos materiales asociados al oxígeno, representado como el elemento básico para la vida. En estos procesos la tercera persona externa ils realiza el proceso material sobre nous, considerado como una agresión, diciendo de forma metafórica que por intereses económicos les privan de lo poco que tienen: la vida, la existencia como derecho inherente de las personas.

Los procesos relacionales definen a las personas representadas, sobre todo al locutor, en términos de lo que son y lo que tienen. Se presentan por tanto las características del locutor evocando el físico: "Bronzé, je l'étais de naissance”, sus bienes materiales, o más bien, la ausencia de éstos: "Sans thune, sandales, sans papiers", y también con circunstancias: "Ce n'est pas au clair de la lune" 9 . En primer lugar, al emplear la categorización por identificación física (van Leeuwen 1996: 54) se enfatiza el color de la piel, un hecho que se presenta en consonancia con las evocaciones metonímicas del rostro y el nombre: "Mon visage est une page qu'on n'arrache pas". Se hace por lo tanto hincapié en este rasgo diferenciador de la persona representada por ser una de las razones que provocan la discriminación. En segundo lugar, la ausencia de bienes materiales evoca la falta de recursos económicos y las situaciones de precariedad en el seno de estas minorías. En último lugar, la circunstancia temporal indica una idealización del espacio que en realidad no existe.

Los procesos mentales de percepción y cognición tienen una presencia importante en la canción Tombés des nues. De esta manera, aparece reflejado con estos procesos lo que se ve: "Les lignes, les stores", y lo que no se conoce: "Je ne connaissais pas l'hiver, je ne savais pas encore". Por su parte, los procesos verbales representan lo que se dice: “Qu'est-ce qu'il y a aujourd'hui au menu?", y lo que no se dice pero se desea escuchar: "Sois le bienvenu!". Con estas expresiones el locutor trata de mostrar la superficialidad de la sociedad y de la política francesa, que muestra su belleza externa, pero que está vacía en el interior. Con ello se pretende decir que sus lemas republicanos basados en la igualdad, la libertad y la fraternidad son para ellos meras palabras vacías de contenido puesto que no consideran que se apliquen de manera real a determinados sectores de la población, como son los inmigrantes. En la canción L'erreur est humaine se expresa también

8. National Association of Securities Dealers Automated Quotation. Se trata de la bolsa de valores electrónica automatizada más grande de EEUU.

9. Es posible que aquí se esté utilizando la intertextualidad con la canción infantil tradicional $A u$ Clair de la Lune. Sin embargo, no se encuentran indicios claros. 
esta crítica al rechazo basado en la identidad cultural y en el grupo de procedencia: "Qui raconte aux enfants que c'est nous les méchants; Il disait: c'est un pays où t'es libre, en gros, enfin selon la couleur de ton chapeau".

En el caso de los procesos verbales, es importante señalar la presencia del discurso indirecto que indica un alejamiento del locutor con lo que dice. De forma paralela, se intenta llamar la atención del destinatario ya que el discurso indirecto que irrumpe en el texto, lo que enfatiza más aún las palabras proferidas (Maingueneau 1991: 141-142): “Qui n'ont dit jamais «allez, viens»; m’entendre dire «va chercher ton or»; m'entendre dire «sois le bienvenu!»". La ironía se crea aquí introduciendo una voz diferente, en este caso, una voz imaginada atribuida a una persona que no existe ni está representada, situada en una posición límite o absurda, y que da entender dos significados contradictorios. Se trata por lo tanto de un mismo enunciado dirigido dos destinatarios diferentes, en este caso, al endogrupo y al exogrupo (Maingueneau 1991: 128, 149-151). De este modo, el locutor muestra las formas de discriminación a las que se ven sometidos los inmigrantes, que al llegar a Francia no se sienten acogidos por los ciudadanos franceses, sino más bien padecen la indiferencia, cuando no rechazo. Perciben que no son bienvenidos y que deben buscar su suerte sin ayuda de aquellos a los que tienen idealizados y consideran que podrían ayudarles.

En la canción Tombés des nues se detecta la presencia continuada de la preposición mais con valor argumentativo (Maingueneau 1991: 238, 239). Maingueneau habla de dos tipos de mais, uno de refutación, por el que se establecería una estructura de diálogo en el interior de un movimiento único de objeción que enlaza la rectificación y la negación. Y otro, el que aparece en esta canción, que sería el de argumentación, que da lugar a dos actos distintos llamados P y Q. Cada uno de ellos conduce a una conclusión llamada $\mathrm{R}$ y no-R. De manera que, de $\mathrm{P}$ surge la conclusión que se denominará $\mathrm{R}$, y el acto llamado Q dará lugar a la conclusión, denominada no-R. En principio, el enunciado presenta $\mathrm{P}$ como la forma de conducir al interlocutor y concluir R. No obstante, no existe a priori fuera del co-texto razón alguna para oponer los enunciados que mais enfrenta, sino que es el propio texto el que por su movimiento instituye esta oposición. Además, según Ducrot (1984: 9) el argumento no-R sería más poderoso e influyente que R. Así en: "Je suis venu et j'ai caressé des vignes, j'attendais le raisin, mais des ces fruits je n'ai vu que les lignes", se podría hacer el siguiente análisis:

$\mathrm{P}$ : Je suis venu et j'ai caressé les vignes. J'attendais le raisin.

$\mathrm{R}$ : El locutor esperaba cosas positivas al llegar.

MAIS 
Q : De ces fruits je n'ai vu que des lignes.

No-R: el locutor no ha encontrado las cosas positivas que esperaba encontrar.

Al considerarse que la conclusión no- $\mathrm{R}$ es más fuerte que $\mathrm{R}$, el resultado final del enunciado es que prima lo negativo sobre lo positivo. Esto significa que tiene más importancia el hecho de no encontrar lo que esperaba que las expectativas, puesto que, podría ser que la premisa de la que se parte, denominada $P$, fuera errónea, por lo que todo lo demás lo sería también. Es decir, puede que la razón por la que el locutor se desilusione sea porque sus expectativas estuvieran demasiado idealizadas con respecto a la realidad. Esta idea se contempla en una referencia que se aparece en el texto: "Et les pieds nus, et la tête dans les nuages", es decir, con este proceso relacional circunstancial el locutor declara de una forma sutil e indirecta que las cosas no eran como él las había pensado pero porque él quizás no las miraba con la atención requerida. Aun así, la preposición mais es una estrategia para enfatizar la buena imagen del locutor, sus aspectos positivos y la idealización equivocada de la imagen de Francia.

La interrogación retórica aparece en "L'erreur est humaine" con el verbo omitido: "L'erreur est humain, mais l'humain?", y deja la interrogación abierta para poder hacer quizás un paralelismo de tipo: "L'erreur est humain, mais l'humain (est) (une) (erreur)?". En este caso la interrogación retórica trata de que la audiencia se vea obligada a reflexionar sobre una situación determinada, y así dirigir el pensamiento del público. En la canción se cuenta que precisamente el locutor es el que ha padecido los errores y las consecuencias del exogrupo, sin embargo, con esta interrogación pretende transmitir una lección de moral en la que el locutor también se inculparía a sí mismo de forma indirecta, puesto que todos, tanto endogrupo como exogrupo y audiencia, se insertan en la categoría de humanos. En todo caso, con el término "humain" se pretende acercar el auditorio al locutor y expresar la crítica a los errores que se cometen contra las personas procedentes de la inmigración.

En relación con los procesos restantes, no aparecen procesos de comportamiento en estas canciones y sólo se detectan tres procesos existenciales que no dan lugar a una interpretación destacable. Uno funciona como un introductor del tópico: "Voici l'histoire"; otro es una interrogación retórica metafórica que pregunta realmente cómo es la situación actual de Francia: "Qu'est-ce qu'il y a aujourd'hui au ménu?", y el tercero es también una metáfora de Francia y de sus carencias, en la línea de la metáfora antes explicada sobre las uvas, aunque en esta ocasión empleando el estereotipo del queso: "Il y a avait pas de quoi faire un fromage au pays du Mont Blanc". Así se confirma la idea de que los procesos materiales, verbales y existenciales son los que contribuyen de forma más adecuada a la enunciación de los 
temas relacionados con la inmigración, el rechazo y la indiferencia de la sociedad francesa ante la inmigración.

\section{Cuestiones finales}

A des niveaux différents, nous avons tous connu le sentiment d'exclusion : se faire refuser l'entrée d'une boîte de nuit, ne pas trouver de logement ou de boulot, avoir la trouille à la vue de la police, bref, se sentir coupable par le seul fait d'exister. C'est la nourriture de notre propos (Perrin 2002).

En este macrotema se utilizan diferentes estrategias discursivas que critican a Francia por considera que no acoge a los inmigrantes a su llegada, que muestra rechazo o indiferencia ante sus problemas, y por ejercer prácticas de lo que se denomina neo-racismo. El locutor de los textos habla de su experiencia individual y se deduce que está ya instalado en Francia. Esta primera persona relata cómo ha vivido la llegada al país y la convivencia con la ciudadanía francesa. Tras la experiencia de llegar a Francia para vivir y trabajar, el locutor reconoce la idealización del país y su sociedad así como la decepción que surge al sentirse ignorado.

Hay que señalar la vocación de estas canciones de construir una memoria histórica gracias a estas estrategias discursivas detalladas. Se configura así un discurso personal y subjetivo en el que se omite información y detalles sobre las personas y los hechos, con la finalidad de convencer a la audiencia de sus propias ideas. Ello hace de estas canciones un discurso puramente subjetivo e ideológico, que llama desde el propio texto a la reflexión sobre las distintas versiones que se pudieran dar de unos mismos hechos.

Las estrategias que se han utilizado, típicas del discurso ideológico, se retoman aquí mostrando una primera persona que destaca sus propios rasgos físicos y su nombre como elemento que le distingue del resto y define su identidad. Ésta se presenta con un alto nivel de especificación y con un papel que podría considerarse en muchos casos de víctima. Por su parte, la tercera persona es la causante de los problemas que padece la primera, apareciendo difusa, sin especificar, sin identificar y marcando una relación de oposición estable y duradera.

Es significativo el paso de lo particular a lo general entre las personas representadas. Esta estrategia trata de captar la atención de una posible audiencia que se identifique con lo que el texto dice, o que pueda entender lo que estas minorías representadas en el endogrupo padecen. El análisis revela igualmente la importancia que se otorga en este macrotema a la representación y el perfil de las personas, 
más que a sus actos. Así, lo que parece importante transmitir para el emisor de forma efectiva es una representación positiva del locutor y del endogrupo, y una representación negativa de la tercera persona ils que se perfila en una relación dialógica entre ambas, en la que el otro no reconoce al locutor en sí mismo y resulta ser la razón fundamental que motiva la discriminación.

A pesar del rechazo, de la injusticia, de la desconsideración social, de las decepciones y de todos los aspectos negativos que van ligados al concepto de racismo y neo-racismo, los textos de las canciones del grupo musical Zebda se muestran animados para crear cambios, y animan de forma explícita a la audiencia a comprometerse en su lucha. Las canciones plantean así la lucha contra la injusticia en un espacio de diálogo multicultural, alegre y libre, con el objetivo de recuperar las cuestiones fundamentales de la dignidad de la persona.

\section{Bibliografía}

ACHUGAR, M. (2008). What we remember. The construction of memory in military discourse. Amsterdam y Philadelphia: John Benjamin.

ADAM, J. M. y BONHOMME, M. (1997). La argumentación publicitaria. Retórica del elogio y de la persuasión. Madrid: Cátedra.

ANDRÉS, R. (2007). Grandes Pensadores. G.W. Hegel. Madrid: Planeta DeAgostini.

AMBROSI, C. y GALLOUX, B. (2004). La France de 1897 à nos jours. París: Armand Colin.

CHERFI, M. (2004). Livret de famille. Arles: Actes Sud.

CHILTON, P. (2004). Analysing Political Discourse. Theory and Practice. Londres: Routledge.

CHIRAC, J. (1991). "Le bruit et l'odeur" < http://www.dailymotion.com/video/ x1ejr5_jacques-chirac-le-bruit-et-l-odeur_news, http://www.youtube.com/ watch?v=eERFYd1DuDE $>$. (Acceso el 4 de noviembre de 2010).

DIJK, T. A. van (1999). Ideología. Una aproximación multidisciplinar. Barcelona: Gedisa.

DIJK. T. A. van (2003). Ideología y discurso: una introducción multidisciplinaria. Barcelona: Ariel.

DUCROT, O. (1984). Decir y no decir. Barcelona: Anagrama.

DUCROT, O. y TODOROV, T. (1972). Dictionnaire encyclopédique des sciences du langage. París: Seuil.

ERBA, S. (2007). Une France pluriculturelle. París: Librio. 
FERRÉOL, G. y JUCQUOIS, G. (2004). Dictionnaire de l'altérité et des relations interculturelles. París: Armand Colin.

GHIO, E. y FERNÁNDEZ, M. D. (2008). Manual de gramática sistémico funcional. El enfoque de M.A.K. Halliday y R. Hasan: aplicaciones a la lengua española. Santa Fe (Argentina): Universidad Nacional del Litoral.

HALLIDAY, M. A. K. (2004). An introduction to Funcional Grammar. Londres: Hodder Education.

KERBRAT-ORECCHIONI, K. (1980). L'énonciation de la subjectivité du langage. París: Armand Colin.

LAKOFF, G. y JOHNSON, M. (1986). Metáforas de la vida cotidiana. Madrid: Cátedra.

LEEUWEN, T. van (1996). "The representation of social actors" en Text and Practices: Reading in critical discourse analysis. (Eds. C.R. Caldas-Coulthard y M. Coulthard). Londres: Routledge: 32-70.

LEFEBVRE, H. (2006). "Enfants de l'inmigration". Manière de voir 19: 57.

LÉVI-STRAUSS, C. (1983). Le regard éloigné. París: Plon.

MAINGUENEAU, D. (1991). L'Analyse du discours. Introduction aux lectures de l'archive. París: Hachette Supérieur.

MARX-SCOURAS, D. (2005). La France de Zebda 1981-2004: Faire de la musique un acte politique. París: Autrement.

MOURA, J.-M. (2010). Le sens littéraire de l'humour. París: Presses Universitaires de France.

NOIRIEL, G. (2006). Le creuset français. Histoire de l'immigration. XIX ${ }^{\text {ème}-X X ~ e ̀ m e ~}$ siècle. París: Seuil.

PERRIN, L. (2002). "Zebda ne retourne pas sa chemise". Libération digital" $<$ http://www.liberation.fr/culture/0101422982-zebda-ne-retourne-pas-sa-che mise>. (Acceso el 6 de julio de 2011).

SEMPRINI, A. (1997). Le multiculturalisme. París: PUF, Coll.

TAPINOS, G. P. (1993). Inmigración e integración en Europa. Barcelona: Fundación Paulino Torras Doménech.

TEMIME, E. (1999). France, terre d'immigration. París: Gallimard.

WINOCK, M. (2003). La France politique. XIX ${ }^{e}-X X^{e}$ siècle. París: Seuil.

YULE, G. (1996). Pragmatics. Oxford: Oxford University Press.

ZEBDA (1992) (grabación sonora). L'arène des rumeurs. Barclay Polygram.

ZEBDA (1995) (grabación sonora). Le Bruit et l'odeur. Barclay Polygram.

ZEBDA (1998) (grabación sonora). Essence Ordinaire. Barclay Polygram.

ZEBDA (2002) (grabación sonora). Utopie d'occase. Barclay Polygram. 